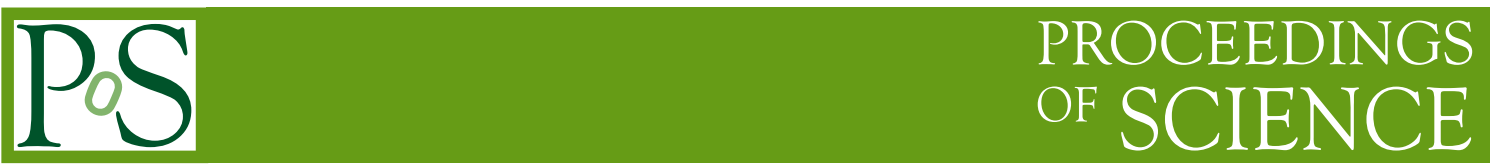

\title{
Ultra-high energy neutrinos: status and prospects
}

\author{
Jaime Alvarez-Muñiz* \\ Departamento de Física de Partículas \& Instituto Galego de Física de Altas Enerxías, \\ Univ. de Santiago de Compostela, 15782 Santiago de Compostela, Spain \\ E-mail: jaime.alvarezmuniz@gmail.com
}

Despite decades of observation, the nature and origin of ultra-high energy cosmic rays (UHECR), with energies in the EeV range and above, remains unknown. The mere existence of UHECR implies that UHE neutrinos should also be produced, but at a level that remains uncertain due to the relatively poor knowledge of some key properties of the sources of UHECR. In the last few years, the IceCube and Pierre Auger observatories have started to constrain potential sources of UHECR through the non-observation of the so-called cosmogenic neutrinos expected to be produced in interactions of UHECR with the Cosmic Microwave Background. The low fluxes predicted in many cosmogenic neutrino models motivate the need for a boost in sensitivity, intended to be achieved with next-generation detectors exploiting the radio technique in dense and dielectric media. Also, existing experiments are planning to improve their performance by building larger and/or more precise detectors. A summary of all these developments will be reported.

35th International Cosmic Ray Conference - ICRC2017

10-20 July, 2017

Bexco, Busan, Korea

${ }^{*}$ Speaker. 


\section{Introduction}

The observation of ultra-high energy cosmic rays (UHECR) of energies above $\sim 10^{18}-$ $10^{20} \mathrm{eV}$ has stimulated much experimental as well as theoretical activity in the field of Astroparticle Physics [1]. It has been experimentally determined that the flux of UHECR above $\sim 4-5 \times 10^{19} \mathrm{eV}$ is suppressed with respect to that extrapolated from lower energies $[2,3,4,5,6,7]$. The position of the break in the energy spectrum is compatible with the Greisen-Zatsepin-Kuz'min (GZK) effect [8], i.e., the interaction of UHECR with the cosmic microwave background (CMB) radiation that limits their propagation distance through the Universe to $\mathscr{O}(100) \mathrm{Mpc}$. However, other scenarios where the limiting energy to which sources can accelerate UHECR is being observed [9] are possible. Key to distinguishing between the different scenarios is the determination of the mass composition of the UHECR [10, 11, 12, 13, 14]. An increasing fraction of primaries heavier than protons is predicted as energy increases in the scenario in which the suppression is due to the sources running out of power to accelerate UHECR [9].

Despite decades of observation, the sources of UHECR remain unidentified. An indication of their extragalactic origin above a few $\mathrm{EeV}$ is the absence of correlation with the Galactic plane of the arrival directions of UHECR as measured in several experiments $[15,16]$. The extragalactic origin was underpinned with the recent observation of a $6.5 \%$ dipolar anisotropy in their arrival distribution in the sky at the Pierre Auger Observatory towards right ascension $\alpha_{d}=100 \pm 10^{\circ}$ and declination $\delta=24_{-13^{\circ}}^{+12^{\circ}}$ [17].

UHE neutrinos are expected to be produced in interactions of UHECR with matter and radiation in the sources of UHECR, and/or in the environment surrounding them, and/or in their propagation through the cosmological radiation backgrounds $[18,19]$ (see Section 2). UHE neutrinos are key messengers in the search for the sources of UHECR. Being neutral, they are not deflected by Galactic or inter-galactic magnetic fields pointing back to sources where they were produced. Being weakly-interacting particles, they can escape the core of dense astrophysical objects and travel unimpeded along cosmological distances, in fact at tens of EeV neutrinos may be the only direct probe of the sources of UHECRs at distances farther than $\sim 100 \mathrm{Mpc}$. UHE neutrinos can also reveal properties of the sources of UHECR such as the evolution of their number density with redshift, not accessible through measurements of the CR spectrum alone [20]. Last but not least, UHE neutrinos can help in the localization of gravitational-wave sources recently detected [21] and enable the study of their astrophysical progenitors.

In Fig. 1 a summary of experimental data on neutrinos is shown. Atmospheric neutrino fluxes produced in the interaction of cosmic rays in the atmosphere are routinely detected by dedicated neutrino observatories such as IceCube [22] and ANTARES [23]. Their fluxes extend in energy up to $\sim 100 \mathrm{TeV}$, well below the UHE regime. A breakthrough in the field of Neutrino Astronomy was the recent detection of an astrophysical neutrino flux, above the atmospheric neutrino background, with the IceCube neutrino observatory. High-energy starting events (HESE) were selected in IceCube by requiring the neutrino interaction vertex to be located inside the fiducial detector volume to reduce the enormous background due to atmospheric muons. The latest astrophysical neutrino spectrum measured in IceCube [24] is also shown in Fig. 1. In six years of data [24], 82 events were detected in an expected background from atmospheric neutrinos of $15.6_{-3.9}^{+11.4}$, representing a $>6 \sigma$ excess, including two neutrinos of energies just above $1 \mathrm{PeV}$, and a $2 \mathrm{PeV}$ event [25]. The 
measured flux is close to the Waxman-Bahcall benchmark flux [26] and is compatible with isotropy which excludes a pure Galactic origin [24]. Also, no point-like sources were identified, mainly due to the fact that most of the detected neutrinos are shower-like for which the angular resolution is relatively poor $\sim 10^{\circ}$.

Above $\sim 10 \mathrm{PeV}$ energy neutrinos have so far escaped observation by existing experiments. These can be detected with a variety of techniques [27] namely, arrays of photo-multiplier tubes buried in ice or under water that observe the Cherenkov light produced by showers or particle tracks induced by neutrinos; arrays of antennas in dense, dielectric media measuring the so-called Askaryan radiation generated in $v$ showers; and particle arrays that sample the front of atmospheric showers induced by $v$. These techniques and the instruments exploiting them will be discussed in Sections 3 and 4.

In this contribution, I highlight some recent developments in the field of UHE neutrinos, with a focus on the current most sensitive experiments and on the most promising techniques and experiments aiming at future neutrino detection in the EeV energy range.

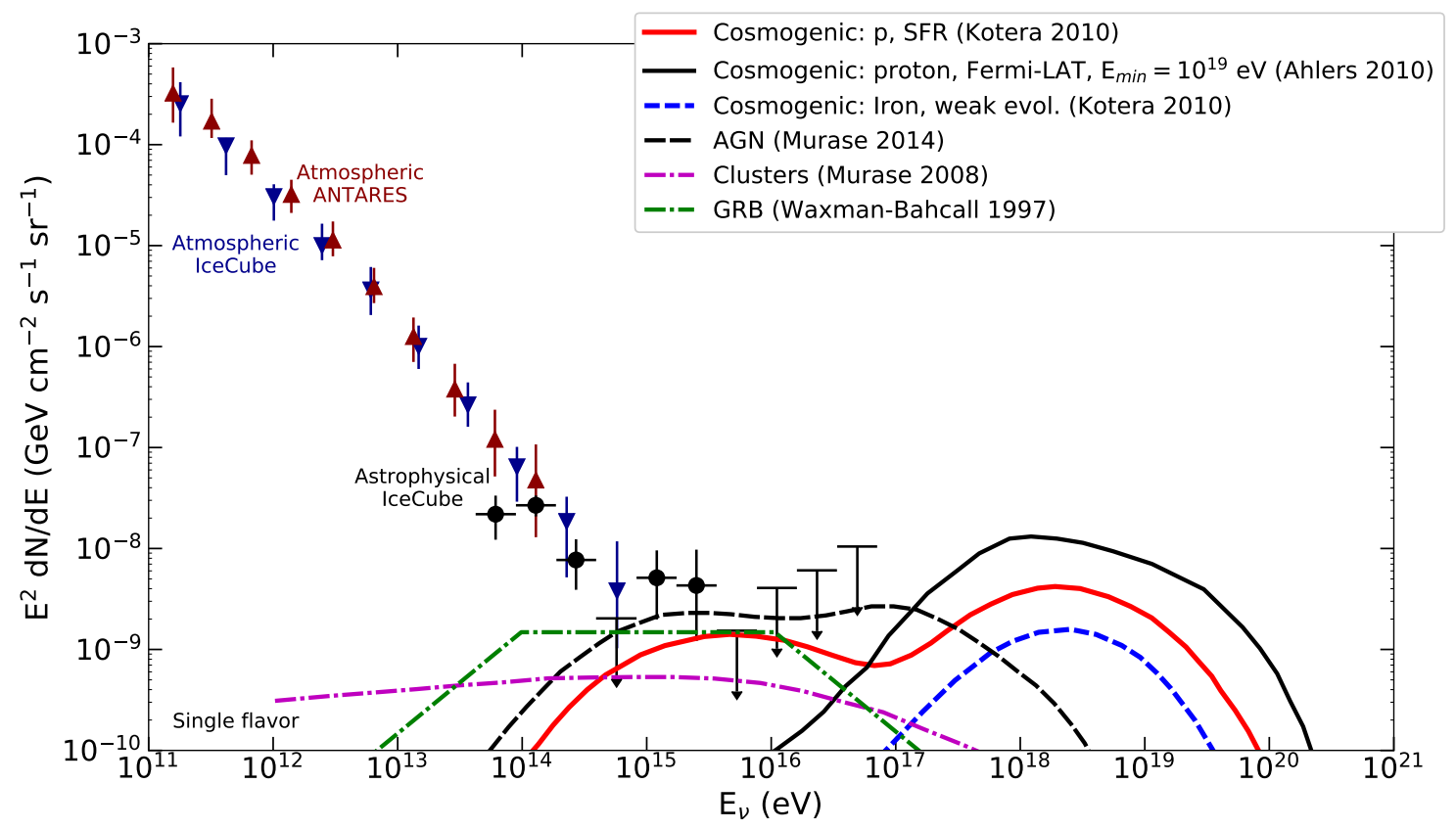

Figure 1: Compilation of experimental data, models of neutrino production in astrophysical sources and cosmogenic neutrino fluxes. Data points with error bars: Atmospheric neutrino fluxes as measured in the IceCube [22] and ANTARES [23] detectors. Also shown is the astrophysical neutrino flux (HESE analysis) measured in IceCube [24]. Lines: Representative examples of astrophysical neutrino model predictions in Active Galactic Nuclei (AGN) [31], Gamma-Ray Bursts (GRB) [33] and Galaxy Clusters [35] (see Section 2.2). Examples of cosmogenic neutrino models from [28] and [37] are also shown (see Section 2.3).

\section{UHE neutrinos: production and potential sources}

\subsection{UHE neutrino production}

In the "standard model" of neutrino production, cosmic rays accelerated to high energy in 
astrophysical sources interact with matter and/or radiation in/at/around the sources, and/or with radiation backgrounds as the CR propagate through the Universe producing neutrinos (see $[18,19]$ for reviews). This mechanism is also responsible for the production of neutrinos in beam-dump man-made accelerators as well as for the production of the fluxes of atmospheric neutrinos (see Fig. 1).

The interaction of $\mathrm{CR}$ with radiation is dominated by the pion photoproduction reaction, where a high-energy proton interacts with a photon with a dominant production of the $\Delta$-resonance that subsequently decays producing a neutron and a charged pion. Charged pions then decay into neutrinos with each of the three neutrinos produced carrying an average energy of $1 / 20$ of the proton energy, while those produced in the decay of the neutron only carry a $\sim 1 / 1000$ of the energy of the parent proton. This process is represented in Eq. (2.1).

$$
p+\gamma \longrightarrow \Delta^{+} \longrightarrow\left\{\begin{array}{l}
n+\pi^{+}\left\{\begin{array}{l}
\pi^{+} \longrightarrow \mu^{+}+v_{\mu} ; \\
n \longrightarrow p+e^{-}+\bar{v}_{e}
\end{array} \mu^{+} \longrightarrow e^{+}+\bar{v}_{\mu}+v_{e}\right. \\
p+\pi^{0} ; \quad \pi^{0} \longrightarrow \gamma+\gamma
\end{array}\right.
$$

The same photoproduction interaction is also responsible for the production of UHE $\gamma$-rays, see Eq. (2.1), providing a direct multi-messenger connection between the fluxes of $\gamma$-rays and neutrinos. Interactions of nuclei heavier than protons with photons can also produce UHE neutrinos. The dominant interaction is photodisintegration of the nucleus leading to a proton that can subsequently photoproduce pions [28].

UHE neutrinos can also be produced in interactions with matter. The reaction, depicted in Eq. (2.2), typically leads to the multiple production of neutrons, pions (and also kaons) that decay into neutrinos and $\gamma$-rays.

$$
p+p \longrightarrow p+n+\pi^{0}+\pi^{ \pm}+\ldots \longrightarrow\left\{\begin{array}{l}
\pi^{+} \longrightarrow \mu^{+}+v_{\mu} ; \mu^{+} \longrightarrow e^{+}+\bar{v}_{\mu}+v_{e} \\
\pi^{-} \longrightarrow \mu^{-}+\bar{v}_{\mu} ; \mu^{-} \longrightarrow e^{-}+v_{\mu}+\bar{v}_{e} \\
n \longrightarrow p+e^{-}+\bar{v}_{e}
\end{array}\right.
$$

Interactions of nuclei heavier than protons can also produce UHE neutrinos through the same type of interactions as those producing neutrinos in $p p$ collisions.

In all the interactions above, the proportion of neutrino flavors is approximately $v_{e}: v_{\mu}: v_{\tau} \simeq$ $1: 2: 0$ at production. Oscillation over the astronomical distances where the sources are thought to be located leads to an approximate equal flavor ratio: $v_{e}: v_{\mu}: v_{\tau} \simeq 1: 1: 1$ [29]. Other scenarios are possible, but they will not be considered in this proceeding (see for instance Appendix B in [19]).

\subsection{Neutrinos produced in astrophysical sources}

Above a few EeV, the sources of UHECR are likely extragalactic [17]. There are many candidate sources of UHECR at cosmological distances where UHE neutrinos can also be produced $[18,19]$. They can be generically classified into two categories: "CR accelerators" and "CR reservoirs" [30]. In CR accelerators UHE $v$ are produced within the source itself, typically through $p \gamma$ 
collisions. Examples are blazars [32] and Gamma-Ray Bursts [33]. In CR reservoirs UHEv are produced in interactions of $\mathrm{CR}$ while they are confined within the environment surrounding the source. Examples of this class are Starburst Galaxies [34], and Galaxy Clusters [35]. Examples of neutrino fluxes predicted in some of these models are shown in Fig. 1. The fluxes typically peak (in an $E^{2} d N / d E$ plot) in the energy range $10^{15}-10^{17} \mathrm{eV}$.

\subsection{Neutrinos produced in the propagation of UHECR through the Universe}

While propagating from their sources to Earth, UHECR can undergo interactions with cosmological radiation backgrounds and give birth to the so-called cosmogenic neutrinos [36, 28]. Protons with energy greater than $E_{p} \sim 5 \times 10^{19} \mathrm{eV}$ are above the threshold for $p \gamma$ interactions with CMB photons producing UHE cosmogenic neutrinos [36], with typical energies around $10^{18} \mathrm{eV}$. Lower energy protons with $E_{p} \sim 10^{18} \mathrm{eV}$ can also interact with photons of energy larger than CMB photons, such as Infrared/Ultraviolet (IR/UV) backgrounds, and produce neutrinos of energies around $10^{16} \mathrm{eV}$. For this reason, cosmogenic neutrino fluxes typically exhibit two peaks in their spectrum. An example taken from [28] is shown in Fig. 1. The height of the peak at $\sim 10^{16} \mathrm{eV}$ depends strongly on the assumed density and evolution with redshift of the IR/UV backgrounds. For instance in [37], also shown in Fig. 1, the contribution to the neutrino flux due to interactions with IR/UV backgrounds is smaller, and only the peak in the spectrum at $\sim 10^{18} \mathrm{eV}$ is apparent.

The fluxes of cosmogenic neutrinos are highly uncertain. At the EeV energies of interest here the shape and normalization of the cosmogenic neutrino flux are very sensitive to the current unknowns of UHECR sources and composition. The fluxes depend mostly on the nature of the UHECR (protons or heavier nuclei), the evolution with redshift $z$ of the unknown sources of UHECR, and on their spectral features at injection (power-law index and maximum proton energy $\left.E_{p \text {,max }}\right)$ [28]. As shown in Figs. 1 and 2 protons typically produce more neutrinos than heavier primaries. Sources with larger comoving number densities in the past produce larger fluxes than sources evolving more weakly with $z$; and sources where UHECR achieve large values of $E_{p \text {,max }}$ produce higher energy neutrinos than sources with low values of that parameter [28, 38]. Observations of UHECR do not uniquely determine these parameters, primarily because interactions of UHECR during propagation obscure the early Universe from direct observation. In this respect, the observation of UHE neutrinos can provide further hints on the dominant scenario of UHECR production, as well as on the evolution with $z$ of their sources which can help in their identification $[20,28]$.

In Fig. 2 we show a range of predictions for cosmogenic neutrino models in the EeV energy range for different assumptions on the UHECR composition and evolution with redshift of the sources. There are many other models in the literature [28, 37], some of them predicting significantly lower fluxes than those shown here [39].

\section{Constraints from IceCube and the Pierre Auger Observatory}

Currently, the three most sensitive neutrino detectors in the energy range above $\sim 10^{17} \mathrm{eV}$ are the IceCube Neutrino Observatory [40], a dedicated neutrino detector; the Pierre Auger Observatory [41], a dedicated UHECR detector that is also sensitive to UHE neutrinos; and the ANtarctic 
Impulsive Transient Antenna (ANITA) instrument [42], a dedicated UHE neutrino detector that has also detected UHECR. The ANITA instrument will be addressed in Section 4.

IceCube is a cubic-kilometer neutrino detector installed in the ice at the geographic South Pole between depths of 1450 and $2450 \mathrm{~m}$, forming a three-dimensional array of digital optical modules attached to strings with a horizontal spacing of $\sim 125 \mathrm{~m}$. The detector construction was completed in December 2010, and the observatory has been in full operation with 86 strings (IC86) since May 2011. During the construction period, it was partially operated with 40, 59, and 79 strings, in 2008-2009, 2009-2010, and 2010-2011, respectively. The analysis described here is based on data taken from April 2008 to May 2017 with an effective lifetime of 3126 days.

Neutrinos are detected in IceCube by observing the Cherenkov light produced in ice by charged particles created when neutrinos interact with the medium and induce muon tracks or cascades depending on flavor and interaction channel (charged-current or neutral-current). The energy spectrum of atmospheric muons and neutrinos falls steeply with energy and the calculated cosmogenic neutrino fluxes, typically having a harder spectra, are expected to dominate over this background in the EeV energy range. This can be seen in Fig. 1. For this reason identification of neutrino candidates in IceCube is done simply by looking for events (showers or tracks) with a large amount of Cherenkov light (EHE analysis [40]) indicative of the fact that the neutrino energy is also large. Three candidates were found in the period from April 2008 to May 2017: one of them turned out to be a spurious event, a flash from a calibration laser [43]. Another event had a deposited energy $E_{\text {dep }} \sim 2.6 \mathrm{PeV}$ and a third event was not fully contained in the fiducial volume of IceCube with an estimated $E_{\mathrm{dep}} \sim 2.72 \mathrm{PeV}$. They are inconsistent with being of cosmogenic origin at $2.8 \sigma$ level [43], and thus they are considered as astrophysical background in searches for cosmogenic neutrinos. Given the absence of neutrinos above $10 \mathrm{PeV}$, a differential limit to the diffuse neutrino flux was obtained [43] as shown in Fig. 2.

A comparable sensitivity to that of IceCube in the EeV energy range is reached with the Surface Detector (SD) of the Pierre Auger Observatory located in Malargüe, Argentina [41]. The SD consists of 1660 water-Cherenkov stations spread over an area of $\sim 3000 \mathrm{~km}^{2}$, which are separated by $1.5 \mathrm{~km}$ and arranged in a triangular grid. The signals produced by the passage of shower particles through the SD detectors are recorded as time traces in $25 \mathrm{~ns}$ intervals.

With the SD of the Pierre Auger Observatory [41], Air showers induced by UHE $v$ can be efficiently identified above $10^{17} \mathrm{eV}$ in the more numerous background of UHECR [46]. Cosmic rays interact shortly after entering the atmosphere and induce extensive air showers whose electromagnetic component gets absorbed due to the large matter depth of atmosphere from the first interaction point to the ground for highly inclined directions. As a consequence, the shower front at ground level is dominated by muons that induce sharp time traces in the water-Cherenkov stations. On the contrary, showers induced by neutrinos at large zenith angles can start their development deep in the atmosphere so that they have a considerable fraction of electrons and photons. These undergo more interactions than muons in the atmosphere, spreading more in time as they pass through the detector. This is also the case for Earth-skimming (ES) showers, mainly induced by tau neutrinos $\left(v_{\tau}\right)$ that traverse horizontally below the Earth's crust, and can interact near the surface inducing a tau lepton that escapes the Earth and decays in flight in the atmosphere above the SD.

The neutrino selection criteria in Auger are summarized in [46]. Deeply-starting downwardgoing showers initiated by neutrinos of any flavor can be efficiently identified for zenith angles 


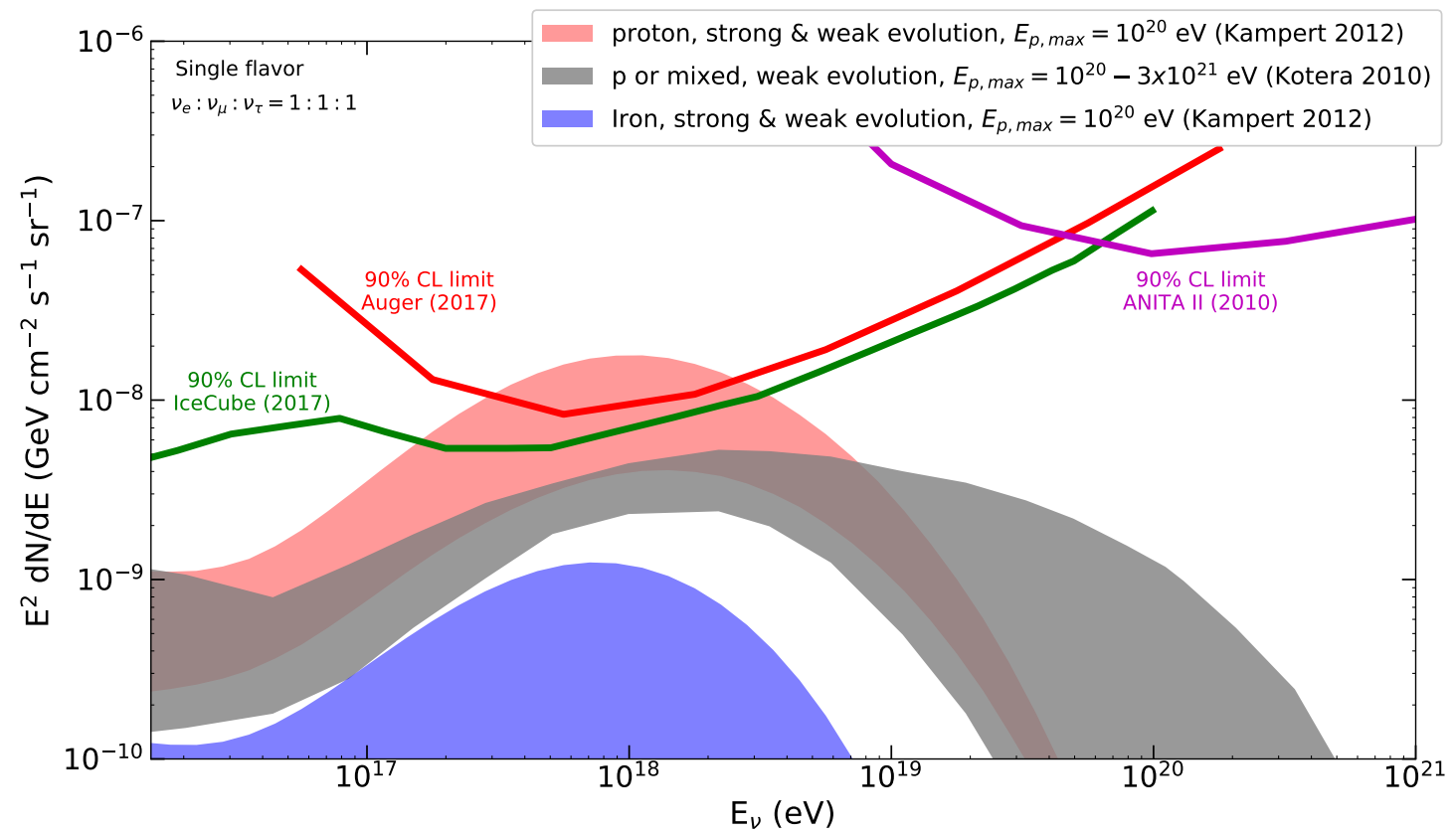

Figure 2: Representative range of cosmogenic neutrino flux predictions depending on the assumed UHECR composition at the sources and their evolution with redshift. Red dashed area: pure proton composition, and strong (following the evolution of Fanaroff-Riley type II (FRII) galaxies) and weak evolutions with redshift (following the Star Formation Rate (SFR)) [44] of the sources of UHECR; gray dashed area: weak source evolutions except uniform and including SFR and GRB-like, for pure protons and a mixed "Galactic" composition [28]; blue dashed area: pure iron at the sources with SFR and FRII evolutions [44]. Also shown in the plot are the world's best limits to the diffuse neutrino flux (differential per decade of energy and converted to single flavor) as obtained from data collected at the IceCube [43] (green solid line), Pierre Auger [45] (red solid line), and ANITA [60] (magenta solid line) observatories.

of $60^{\circ}<\theta<90^{\circ}$ using an optimized cut in a single discrimination variable related to the time spread of the signals in the water-Cherenkov stations. For the ES channel typically only $v_{\tau}$-induced showers with zenith angles $90^{\circ}<\theta<95^{\circ}$ can trigger the SD. This channel is the most sensitive to UHE neutrinos, mainly due to the larger grammage and higher density of the target (the Earth) where neutrinos are converted, and where tau leptons can travel tens of kilometers. Applying these criteria a search for ES as well as DG neutrino-induced showers was performed in Auger data in the period from January 2004 to March 2017 with no neutrino candidates identified. Based on this non-observation a differential limit to the diffuse flux of UHE neutrinos was obtained [45] as shown in Fig. 2.

The IceCube and Auger differential limits are at a comparable level in the EeV energy range. The large area of the SD and the efficient conversion of Earth-skimming $v_{\tau}$ into showers partly compensates the small solid angle where ES showers can trigger the SD. On the other hand IceCube has a relatively small area of $\sim \mathrm{km}^{2}$ but embedded in a dense material enhancing neutrino interactions. At $\mathrm{EeV}$ energies, neutrinos in IceCube can be viewed from a wide range of solid angles typically above the detector (i.e., downward-going from the point of view of IceCube). Moreover, neutrino-induced muons and tau leptons have ranges of $\mathscr{O}(10) \mathrm{km}$ at these energies 
[47] increasing the effective volume for neutrino interactions. Both observatories have started to constrain models of cosmogenic neutrino production that assume pure protons at the sources with strong evolution (FRII) with redshift. An example is the upper edge of the red shaded area [44] in Fig. 2, for which $\sim 10(\sim 6)$ events per lifetime are expected in IceCube (Auger) while none is seen, excluding that flux model at more than $90 \% \mathrm{CL}$. In contrast, at least a 10-fold increase in the current IceCube or Auger exposure will be needed to reach the most optimistic predictions of cosmogenic neutrino flux predictions at $10^{18}-10^{19} \mathrm{eV}$ if the primaries are pure iron. This is out of the range of the current configuration of both observatories. Recent measurements with the Auger Observatory [10, 12] and the Telescope Array [11, 13] (see also [14]) disfavor a significant iron fraction in the cosmic-ray composition at energies up to $4 \times 10^{19} \mathrm{eV}$, and this, in turn, favors higher fluxes of cosmogenic neutrinos. Despite of this, there is a clear need for a significant boost in sensitivity to be able to constrain or detect these flux levels.

\section{Radio detectors}

To achieve sensitivity to the full range of cosmogenic neutrino models shown in Fig. 2, a different detection mechanism is needed that allows instrumenting larger volumes than those monitored by IceCube and the Pierre Auger Observatory at a comparable or if possible smaller cost.

One such possibility that has received a great deal of attention from the theoretical, as well as experimental points of view in the last 25 years is the so-called radio technique (see $[48,49,50]$ for recent reviews). Neutrino telescopes that utilize this technique search for coherent, broadband and impulsive radio signals that are emitted by electromagnetic particle cascades induced by neutrinos interacting in a dense, dielectric and transparent medium. The mechanism of emission of coherent electromagnetic radiation is the so-called Askaryan effect [51]. This is the "entrainment" of electrons from the medium in the shower flow as the shower evolves, and it is mainly due to $\mathrm{MeV}$ shower photons and electrons interacting with atomic electrons through Compton, Moeller and Bhabha processes. As a consequence, a $\sim 20-25 \%$ electron excess develops in the otherwise neutral shower [51,52] avoiding the cancellation of the electric fields due to positive and negative charges. Annihilation of positrons in the shower with electrons in the medium also contributes to this excess by removing positive charges but to a lesser extent than the other processes [52]. For wavelengths longer than the typical dimensions of the shower along the direction perpendicular to the shower axis, the signal is emitted coherently and the electric field strength is proportional to the excess charge and in turn to the shower energy. This occurs in a dense medium such as ice for frequencies up to $\sim 1 \mathrm{GHz}$ because the transverse dimensions of the shower are of the order of 10 $\mathrm{cm}$. Since the charge excess is moving at a velocity greater than the phase velocity of light in the medium, the induced radiation exhibits Cherenkov-like characteristics such as strong emission in a cone of opening angle $\theta_{\text {Cher }}$ determined by the refractive index $n$ of the medium (for instance in ice $\left.n \sim 1.78, \theta_{\text {Cher }} \sim 56^{\circ}\right)$. The Askaryan effect was first observed in a beam test at SLAC National Accelerator Laboratory [53] using a target of silica sand and later in other dielectric media including salt and ice [54], confirming the expected coherent, broadband and impulsive characteristics of the radiation $[52,55]$.

One of the advantages of the radio technique besides its coherent properties is that large naturally-occurring volumes of dense, dielectric and radio-transparent material exist, most promi- 
nently and widely used the Antarctic ice, with attenuation lengths $\mathscr{O}(1) \mathrm{km}$ at hundreds of $\mathrm{MHz}$ frequency $[48,49]$. This fact added to the cost-effectiveness of detectors (antennas) make the radio technique the best candidate for instrumenting a volume of $\mathscr{O}(100) \mathrm{km}^{3}$.

The radio technique can be realized in several ways as sketched in Fig. 3. A ballon-borne detector, such as ANITA [42] or the Exa-Volt Antenna (EVA) [56], far from the neutrino interactions (altitude $\sim 37 \mathrm{~km}$ ) has the disadvantage of a high energy threshold because the radio signals attenuate with distance. Also it has a total lifetime limited to $\sim 1$ month. On the other hadn, these are partly compensated by the fact that being at a high altitude it can view a much larger area of ice compared to an experiment on the ground. Detectors on the ground, such as the Antarctic Ross Ice Shelf Antenna Neutrino Array (ARIANNA) [57], or embedded in their detection medium, such as the Askaryan Radio Array (ARA) [58], have a lower energy threshold than balloon-borne experiments and, in addition, the lifetime can be much longer (years compared to $\sim 1$ month). The drawback of being so close to the interactions is that a smaller volume of ice can be monitored by each detector element which calls for an array of antennas covering a large area.

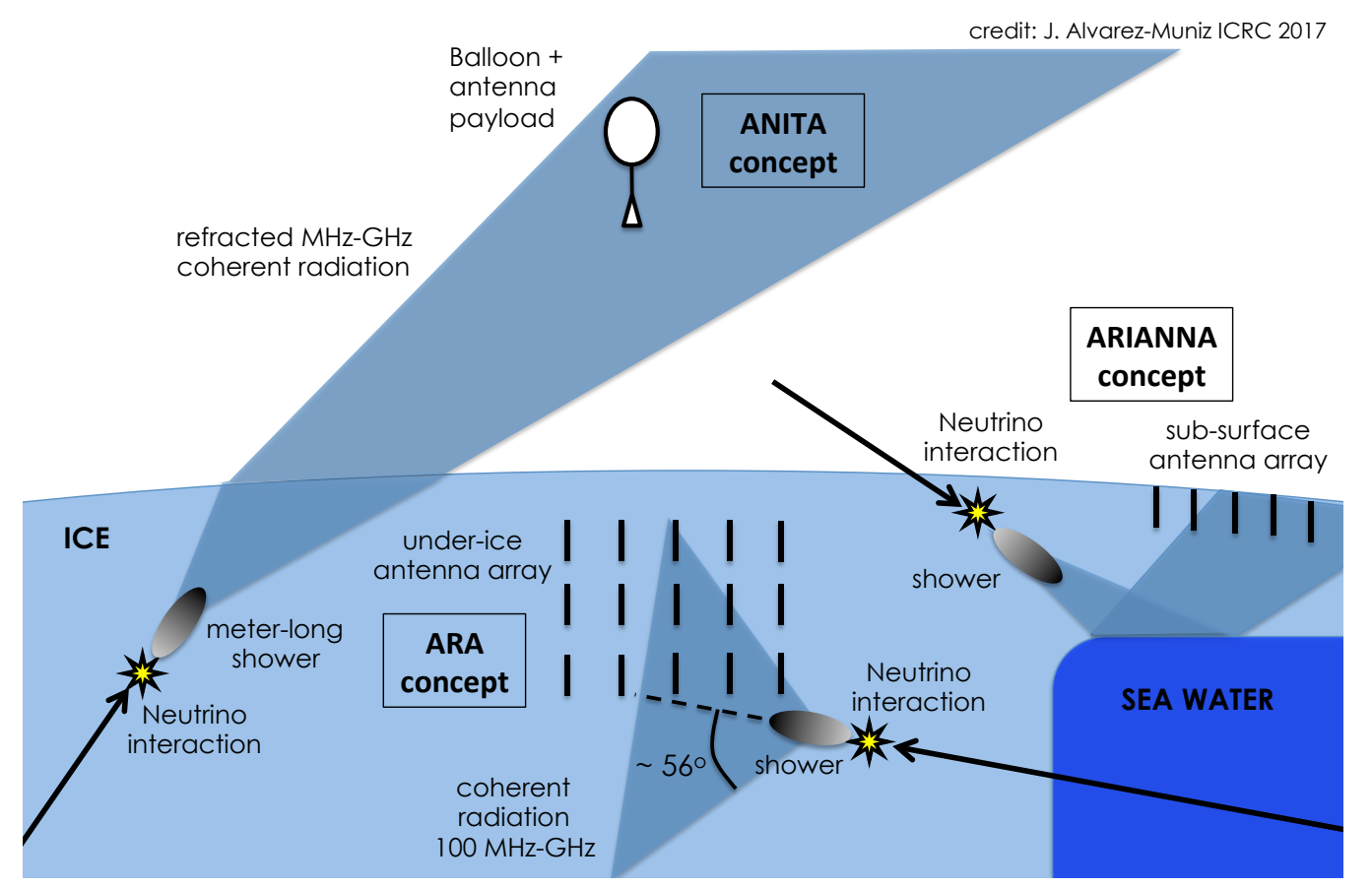

Figure 3: Sketch of the concepts in which different existing, under-construction and planned neutrino observatories are exploiting the radio technique in ice: balloon-borne instruments monitoring a large volume of ice (ANITA [42] and EVA [56]), and arrays of antennas near the surface (ARIANNA) [57], or buried under the ice (ARA) [58]. See text for more details on these experimental initiatives.

ANITA is a balloon-borne instrument that flew at $37 \mathrm{~km}$ altitude over the Antarctic continent carrying a payload of dual-polarized quad-ridged horn antennas with 200-1200 MHz bandwidth to search for impulsive, broadband and predominantly vertically polarized signals from neutrinos 
interacting in the ice sheet [42] (see the sketch in Fig. 3). The instrument can view an area of $\sim 1.5 \times 10^{6} \mathrm{~km}^{2}$. ANITA has currently flown four different instruments: ANITA-I was launched in December 2006, ANITA-II in 2008/09, ANITA-III in 2014, and ANITA-IV in 2016 [59]. ANITA searched for isolated signals from the ice, rejecting thermal noise and anthropogenic backgrounds. The analysis of ANITA-II data yielded one candidate compatible with background expectations and produced the world's best limit on the neutrino flux at energies above $3 \times 10^{19} \mathrm{eV} \mathrm{[60]} \mathrm{shown}$ in Fig. 2. ANITA-II flew for 31 days which was a relatively long flight, but not enough to constrain even the most optimistic fluxes of cosmogenic neutrinos shown in Fig. 2.

Building on ANITA's experience the EVA project was proposed to transform the balloon itself into a high-gain toroidal reflector antenna [56]. The increase in antenna gain compared to ANITA is expected to reduce the neutrino energy threshold by a factor 10. The whole idea of EVA is possible due to the use of super-pressure balloons where the inside of the balloon is kept at a higher pressure than the outside ambient pressure, keeping its shape nearly unchanged over a flight. A prototype balloon was instrumented with a reflector receiver system [56], and the feasibility of the idea was demonstrated. A 3-year sensitivity assuming a 30-day flight per year is shown in Fig. 4. The improve in sensitivity and lower energy threshold when compared to ANITA is apparent.

ARIANNA is a ground-based array of radio antennas on the Ross Ice Shelf in Antarctica [57]. The goal is to deploy a $36 \times 36$-detector array spread over an area of more than $10^{3} \mathrm{~km}^{2}$ buried near the surface of the ice with no drilling needed. Each element of the array consists of several high-gain log-periodic dipole antennas sensitive in the frequency range from $80 \mathrm{MHz}$ to $1.3 \mathrm{GHz}$ downward-pointing. At the bottom of the Ross Ice Shelf at about $600 \mathrm{~m}$ depth, there is a reflecting layer of ocean water where radio signals from downward-going neutrinos interacting in the ice reflect back to the surface (see sketch in Fig. 3). This increases the solid angle for neutrino detection, which in addition to the large surface planned to be covered with antennas, makes ARIANNA a very sensitive neutrino detector in the EeV range. The 3-year projected sensitivity is plotted in Fig. 4, where a duty cycle of 58\% per year is assumed if the array is powered by solar panels with batteries. A factor of $\sim 2$ improvement is expected if a wind-power solution is achieved. Cosmogenic models assuming pure proton and even mixed composition would be severely constrained by ARIANNA in three years of data taking if no events are found. The ARIANNA Collaboration has already deployed eight prototype stations some of them have been working since 2013. Ice properties can strongly influence the final layout of the ARIANNA array [61].

The Askaryan Radio Array (ARA) is a proposed 37-station array of receiver antennas embedded in the ice near the South Pole at $200 \mathrm{~m}$ depth covering an area of $\sim 100 \mathrm{~km}^{2}$ [58]. Each station consists of a mixture of 16 antennas to measure the horizontal and vertical polarizations and calibration equipment deployed along strings. The 3-year sensitivity of the proposed ARA-37 array is shown in Fig. 4 and is similar to ARIANNA sensitivity in the EeV range despite the smaller instrumented area of ARA and partly due to the 58\% duty cycle assumed for ARIANNA, and to the larger attenuation length of ice at the South Pole compared to the one at the Ross Ice Shelf $[49,61]$. A Testbed prototype ARA station was deployed in the 2010/11 season at $30 \mathrm{~m}$ depth, followed by three stations, with three more to be deployed in the 2017/18 season. One of this stations will include a phased-array of antennas to achieve a high signal-to-noise ratio to lower the energy threshold for neutrino detection [62].

An interesting possibility is to search for Askaryan radio emission from neutrino interactions in 


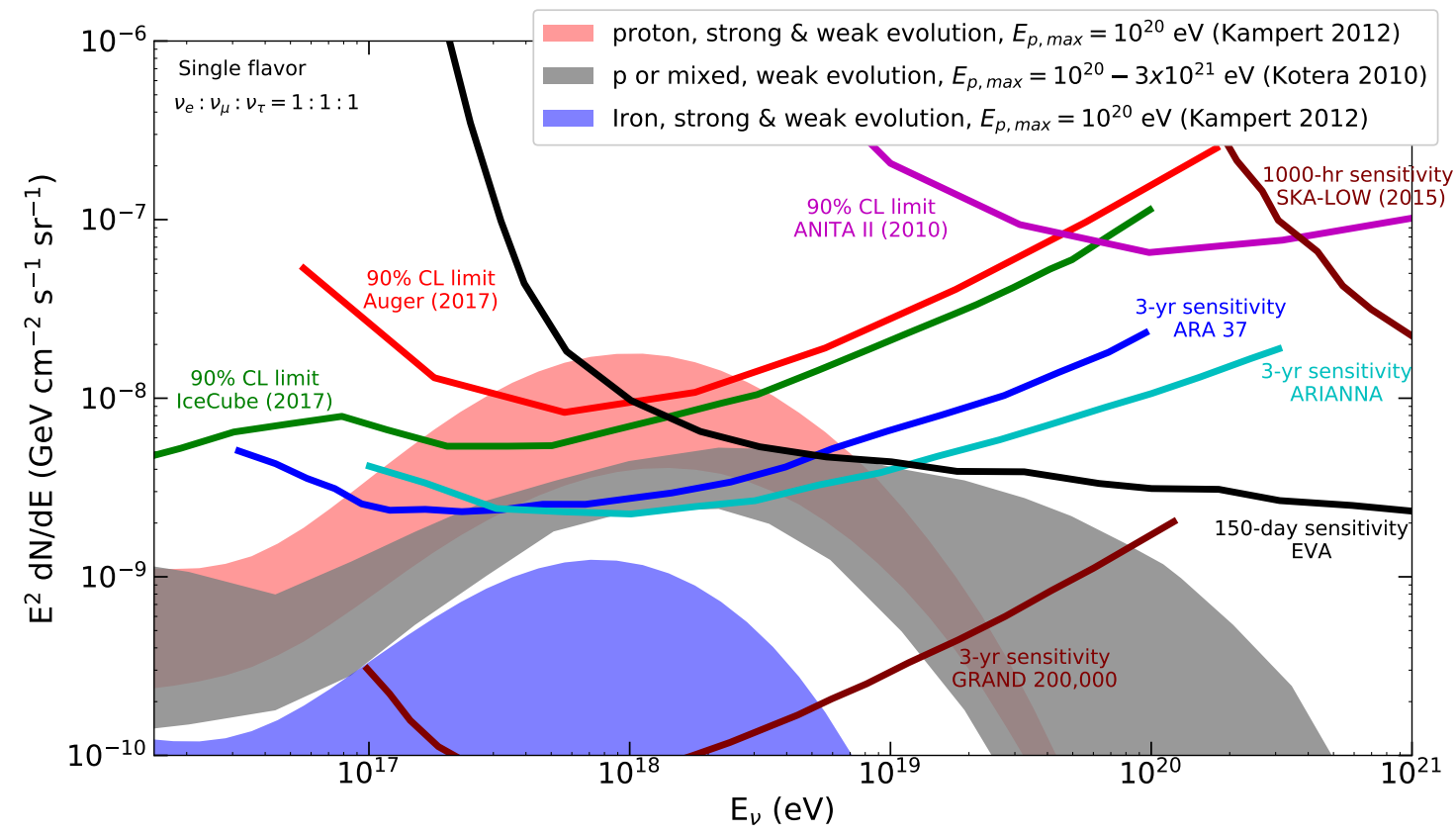

Figure 4: A compilation of projected sensitivities of various experiments exploiting the radio technique. Black line: EVA [56]; cyan line: ARIANNA [57]; blue line: ARA [58]; dark red line: GRAND [63]; brown line: SKA-LOW Phase 1 [64]. For comparison, the differential limits in Fig. 2 are also shown. All limits and sensitivities are given for single flavor and per decade of energy. See caption of Fig. 2 for details on the cosmogenic neutrino flux model predictions.

the regolith of the Moon with existing radiotelescopes operating in the $100 \mathrm{MHz}$ to a few $\mathrm{GHz}$ frequencies. Radio frequency interference can be reduced by requiring signals in different frequency bands to exhibit the characteristic delays due to dispersion in the ionosphere. Many initiatives and experiments have already searched for these lunar pulses (see [64] for a comprehensive review). The planned Square Kilometer Array (SKA) will be more sensitive to neutrinos interaction in the moon than any of the existing lunar experiments due to its large collecting area. Construction of Phase 1 of SKA is scheduled to begin in 2018. The sensitivity after 1000 hours of observation in the frequency band 50-350 MHz (SKA-LOW1) [65] is shown in Fig. 4. The drawback is that neutrino interactions occur near the surface of the Moon at a distance of about one light-second and this induces a very high energy threshold for neutrino detection of $\sim 10^{20} \mathrm{eV}$.

\subsection{Radio emission from air showers induced by tau neutrino interactions in Earth}

A great deal of attention is being paid to the possibility of UHE tau-neutrino detection $\left(v_{\tau}\right)$. An observation of a tau-neutrino interaction would be a milestone in Neutrino Astronomy. UHE $v_{\tau}$ are not expected to dominate the neutrino flux at production, and hence their appearance will be a strong indication for oscillation over astronomical distances.

At EeV energies the detection probability of $v_{\tau}$ is expected to be enhanced by the efficient conversion of neutrinos into $\tau$-leptons in the Earth crust (see for instance [66] and refs. therein). $\tau$-leptons at EeV energy travel tens of $\mathrm{km}$ in rock and can exit the Earth and decay in the atmosphere 
producing atmospheric showers. These can be detected either with particle arrays, as is the case for the SD of the Pierre Auger Observatory, or with arrays of radio detectors on the ground or balloonborne instruments such as ANITA (see sketch in Fig. 5). The charged particles in the atmospheric shower resulting from the $\tau$ decay generate radio emission in part due to the geomagnetic effect: positively and negatively charged particles are split due to the Earth's magnetic field, yielding coherent emission. Askaryan radiation is also emitted from the shower, but this mechanism is subdominant in air showers for most geometries.

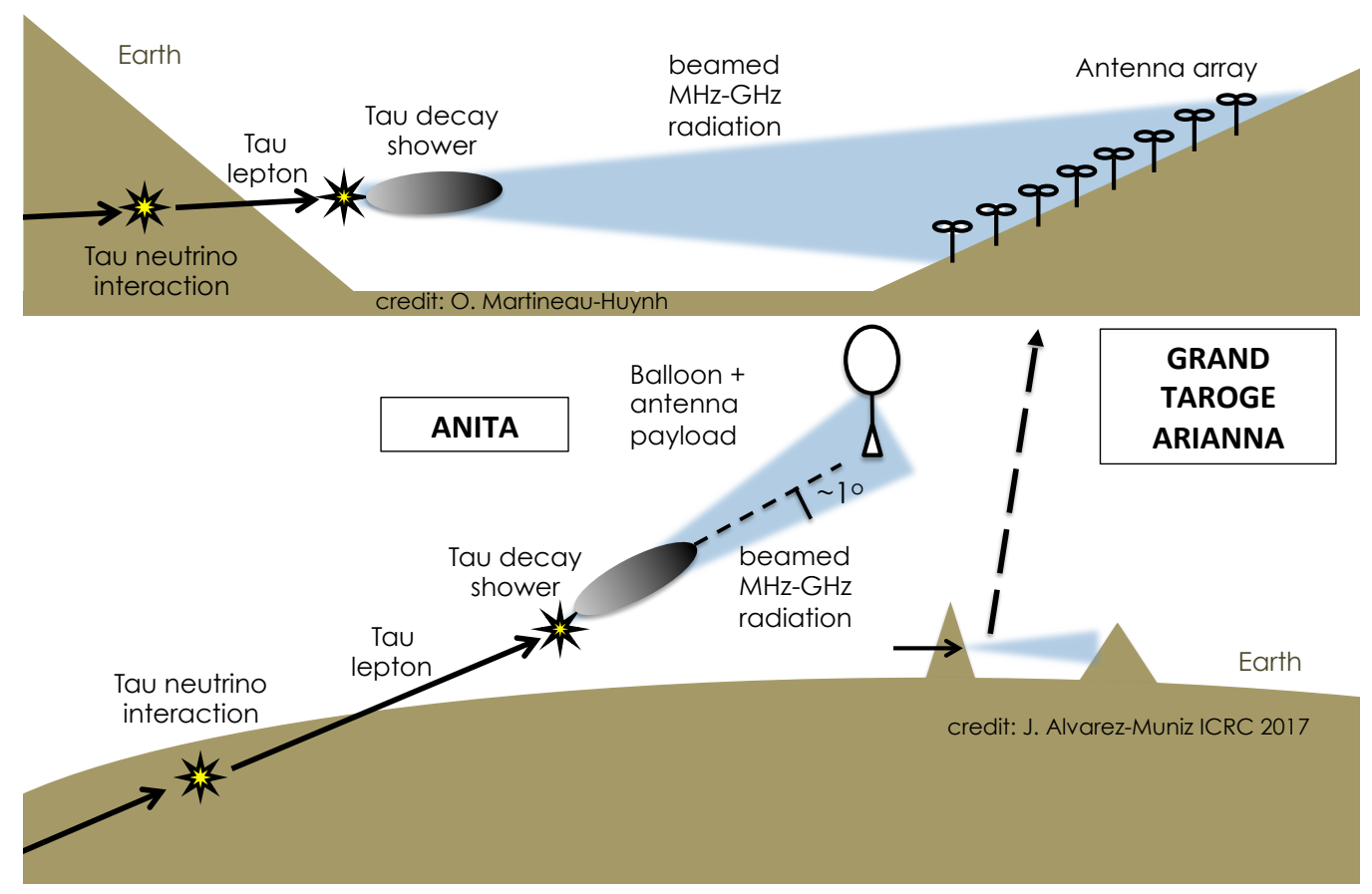

Figure 5: Sketch of the different concepts for UHE $\tau$ neutrino detection.

An intriguing event was recently detected in the data collected during the ANITA-II flight in a search for radio emission from showers induced by UHECR [67]. The event arrived at the payload with an angle $\sim 27.4^{\circ}$ below the horizontal. This fact immediately excluded "direct" emission from a CR atmospheric-skimming shower seen above the horizon and not crossing the Earth's crust. Also, the radiation is strongly horizontally polarized excluding the possibility that it was originated in a neutrino interaction under ice for which mostly vertically polarized signals are expected [52]. Also, the possibility that this was radiation induced in a downward-going CR shower reflected on the ice cap and triggering the ANITA instrument is excluded since an inversion of the horizontal polarization at reflection is expected and this was not seen. Anthropogenic origin of the event is also strongly disfavored at $3 \sigma$ although not completely excluded. The main difficulty in interpreting the event as an upward-going $v_{\tau}$ lies in the fact that the required flux to explain it is in strong tension with Auger and IceCube upper limits at EeV energies shown in Fig. 2, see [68]. 
The event is still under investigation. Data analysis of ANITA-IV data is in progress and might shed further light on this intriguing event, especially if another event alike shows up in data.

Recently the Giant Radio Array for Neutrino Detection (GRAND) experiment has been proposed to detect radio emission from atmospheric showers induced by the decay of $\tau$ leptons created in the interaction of neutrinos under the Earth's surface [63] (see sketch in Fig. 5). GRAND plans to build an enormous detector consisting of an array of $\sim 10^{5}$ radio antennas operating in the $60-200$ MHz band deployed over $2 \times 10^{5} \mathrm{~km}^{2}$ in a mountainous area in China. The aim of GRAND is to reach a single-flavor sensitivity of $E^{2} d N / d E \sim 5 \times 10^{-11} \mathrm{GeV} \mathrm{cm}^{2} \mathrm{~s}^{-1} \mathrm{sr}^{-1}$ above $5 \times 10^{17} \mathrm{eV}$, well beyond the reach of other planned detectors. The 3-year projected sensitivity of GRAND is shown in Fig. 4 probing well below the flux predictions of cosmogenic neutrinos from UHECR iron primaries. Currently, the deployment of a prototype (GRANDproto) is underway [69]. This is a hybrid detector composed of 35 antennas and 24 scintillators at the site of the pioneering TREND experiment [70] in the Tianshan mountains. Other experiments exploiting the same idea are TAROGE in Taiwan looking down from a mountain to the ocean [71], and a planned array of antennas in the area around the Transantarctic Mountains in Antarctica [72].

\section{Summary and outlook}

We have just witnessed the beginning of a new era in Neutrino Astronomy with the detection of up to a few PeV neutrinos with the IceCube Observatory, and we hope to witness the dawn of UHE $\left(>10^{17} \mathrm{eV}\right)$ Neutrino Astronomy.

IceCube and the Pierre Auger Observatory are currently the most sensitive experiments in the energy range around $10^{18} \mathrm{eV}$. The non-detection of UHE neutrinos by these two experiments has allowed us to place constraints on the potential sources of UHECR, those where proton production dominates and have a strong evolution with redshift are disfavored as the solution to the longstanding problem of the origin of the UHECR. ANITA-II remains the most-sensitive instrument above $3 \times 10^{19} \mathrm{eV}$.

Neutrino detectors based on the observation of Askaryan radiation in dense, dielectric media can provide the needed boost in sensitivity with respect to current experiments. The in-construction ARA, ARIANNA, and EVA can provide a 10-fold increase in sensitivity. The detection of $v_{\tau}$ is a promising possibility with ambitious proposals such as the GRAND antenna array aiming at a 100 -fold boost to be sensitive to some of the most pessimistic models of cosmogenic neutrino production.

The possibility that UHE neutrinos are detected very soon cannot be neglected, although most likely detection will need to wait longer. Once the initial discovery is made, it will open up a unique window to the Universe at the highest energies.

\section{Acknowdlegments}

J. A-M is supported by Ministerio de Economía (FPA 2015-70420-C2-1-R); Consolider Ingenio 2010 CPAN Programme (CSD2007-00042), Xunta de Galicia (GRC2013-024); Feder Funds, $7^{\text {th }}$ Framework Program (PIRSES-2009-GA-246806); and RENATA Red Nacional Temática de Astropartículas (FPA2015-68783-REDT), Spain. I thank the organizers of the ICRC 
2017 for the kind invitation to give a highlight talk on the exciting topic of UHE neutrinos. I also thank L. A. Anchordoqui, D. Besson, S. W. Barwick, P. Billoir, J. D. Bray, W. R. Carvalho, K. Fang, P. L. Ghia, A. Ishihara, C. W. James, K.-H. Kampert, A. Karle, D. García-Fernández, O. Martineau-Huynh, G. Parente, F. Pedreira, R. Piegaia, P. Pieroni, A. Romero-Wolf, E. Roulet, M. Schimp, O. Scholten, H. Schoorlemmer, D. Seckel, T. Stanev, M. Trini, M. Tueros, M. Unger, R. A. Vázquez, A. van Vliet, A. G. Vieregg, A. A. Watson, E. Waxman, L. Yang, S. Yoshida and E. Zas for various enlighting discussions on this topic in the last few years.

\section{References}

[1] A. A. Watson, Rep. Prog. Phys. 77, 036901 (2014), and refs. therein.

[2] R. U. Abbasi et al. [HiRes Collaboration], Phys. Rev. Lett. 100, 101101 (2008).

[3] J. Abraham et al. [Pierre Auger Collaboration], Phys. Rev. Lett. 101, 061101 (2008); J. Abraham et al. [Pierre Auger Collaboration], Phys. Lett. B 685, 239 (2010).

[4] T. Abu-Zayyad et al. [Telescope Array Collaboration], ApJ 768, L1 (2013).

[5] F. Fenu, for the Pierre Auger Collaboration, PoS(ICRC2017)486.

[6] Y. Tsunesada, for the Telescope Array Collaboration, PoS(ICRC2017)535.

[7] D. Ivanov, for the Telescope Array and on behalf of the Pierre Auger Collabs. PoS(ICRC2017)498.

[8] K. Greisen, Phys. Rev. Lett. 16, 748 (1966);

G. T. Zatsepin and V. A. Kuzmin, Pis'ma v Zhurnal Eksperimental'noi i Teoreticheskoi Fiziki 4, 114 (1966), JETP Lett. 4, 78 (1966) (Engl. Transl.).

[9] D. Allard, Astropart. Phys. 33, 39 (2012).

[10] P. Abreu et al. [Pierre Auger Collaboration], Phys. Rev. Lett. 104, 091101 (2010); A. Aab et al. [Pierre Auger Collaboration], Phys. Rev. D 90, 122005 (2014).

[11] R. U. Abbasi et al. [Telescope Array Collaboration], Astropart. Phys. 64, 49 (2015).

[12] J. Bellido and for the Pierre Auger Collaboration PoS(ICRC2017)506.

[13] W. Hanlon for the Telescope Array Collaboration, PoS(ICRC2017)536.

[14] V. De Souza, for the Pierre Auger Collab. and on behalf of the Telescope Array Collab., PoS(ICRC2017)522.

[15] A. Aab et al. [Pierre Auger Collaboration], Astrophys. J. 804, 15 (2015).

[16] S. Troitsky, for the Telescope Array Collaboration, PoS(ICRC2017)548.

[17] A. Aab et al. [Pierre Auger Collaboration], Science 357, 1266 (2017).

[18] J. K. Becker, Phys. Rep. 458, 173 (2008) and refs. therein.

[19] L. A. Anchordoqui et al., Journal of High Energy Astrophysics, 1, 1 (2014) and refs. therein.

[20] D. Seckel and T. Stanev, Phys. Rev. Lett. 95, 141101 (2005).

[21] B. P. Abbott et al. [LIGO Scientific Collaboration, Virgo Collaboration], Phys. Rev. Lett. 116, 061102 (2016); Phys. Rev. Lett. 116, 241103 (2016); Phys. Rev. Lett. 118, 221101 (2017).

[22] M. G. Aartsen et al. [IceCube Collaboration], Eur. Phys. J. C 75, 3, 116 (2015).

[23] S. Adrián-Martínez et al. [ANTARES Collaboration], Eur. Phys. J. C 73, 2606 (2013). 
[24] C. Kopper for the IceCube Collaboration, PoS(ICRC2017)981.

[25] M. G. Aartsen et al. [IceCube Collaboration], Phys. Rev. Lett. 113, 101101 (2014) and Supplementary Methods and Tables.

[26] E. Waxman and J. Bahcall, Phys. Rev. D 59, 023002 (1999).

[27] B. Baret and V. Van Elewyck, Rep. Prog. Phys. 74, 046902 (2011) and refs. therein.

[28] K. Kotera, D. Allard, and A. Olinto, JCAP 10, 013 (2010), and refs. therein.

[29] H. Athar, M. Jeżabek and O. Yasuda, Phys. Rev. D 62, 103007 (2000).

[30] K. Murase and E. Waxman, Phys. Rev. D 94, 103006 (2016) and refs. therein.

[31] K. Murase, Y. Inoue, and C.D. Dermer, Phys. Rev. D 90, 023007 (2014).

[32] K. Mannheim, Astropart. Phys. 3, 295 (1995); A. Atoyan and C. D. Dermer, Phys. Rev. Lett. 87, $221102(2001)$.

[33] E. Waxman and J.N. Bahcall, Phys. Rev. Lett. 78, 2292 (1997).

[34] A. Loeb and E. Waxman, J. Cosmol. Astropart. Phys. 05, 003 (2006).

[35] K. Murase, S. Inoue, and S. Nagataki, Astrophys. J. Lett 689, L105 (2008).

[36] V. S. Berezinsky and G. T. Zatsepin, Phys. Lett. B 28, 423 (1969).

[37] M. Ahlers et al., Astropart. Phys. 34, 106 (2010).

[38] A. van Vliet, R. Alves Batista and J. Hörandel, PoS(ICRC2017)562.

[39] A. M. Taylor, M. Ahlers and D. Hooper, Phys. Rev. D 92, 063011 (2015).

[40] M. G. Aartsen et al. [IceCube Collaboration], Phys. Rev. D 88, 112008 (2013).

[41] A. Aab et al. [Pierre Auger Collaboration], Nucl. Instrum. Methods Phys. Res., Sect. A 798, 172 (2015).

[42] P. W. Gorham et al. [ANITA Collaboration], Astropart. Phys. 32, 10 (2009).

[43] S. Yoshida for the IceCube Collaboration, PoS(ICRC2017)975.

[44] K.-H. Kampert, M. Unger, Astropart. Phys. 35, 660 (2012).

[45] E. Zas for the Pierre Auger Collaboration, PoS(ICRC2017)972.

[46] A. Aab et al. [Pierre Auger Collaboration], Phys. Rev. D 91, 092008 (2015).

[47] S. I. Dutta, M.H. Reno, I. Sarcevic and D. Seckel, Phys. Rev. D 63, 094020 (2001).

[48] A. L. Connolly and A. G. Vieregg, in "Neutrino Astronomy Current Status, Future Prospects", World Scientific, 2017. arXiv:1607.08232v1 [astro-ph.HE] and refs. therein.

[49] T. Huege and D. Besson to appear in Progress of Theoretical and Experimental Physics (2017). arXiv:1701.02987v1 [astro-ph.IM] and refs. therein.

[50] F.G. Schröder, Progress in Particle and Nuclear Physics 93, 1 (2017).

[51] G. A. Askaryan, Sov. Phys. JETP 14, 441 (1962); 48, 988 (1965).

[52] E. Zas, F. Halzen and T. Stanev, Phys. Rev. D 45, 362 (1992).

[53] D. Saltzberg et al., Phys. Rev. Lett. 86, 2802 (2001). 
[54] P. W. Gorham et al., Phys. Rev. D. 72, 023002 (2005). P. W. Gorham et al., Phys. Rev. Lett. 99, 171101 (2007)

[55] J. Alvarez-Muñiz, A. Romero-Wolf, E. Zas, Phys. Rev. D 81, 123009 (2010).

[56] P. W. Gorham et al., Astropart. Phys. 35, 242 (2011); A. Romero-Wolf et al., PoS (ICRC 2015) 1151.

[57] S. W. Barwick et al. [ARIANNA Collaboration], Astropart. Phys. 70, 12 (2015); Christopher Persichilli for the ARIANNA Collaboration, PoS(ICRC2017)977.

[58] P. Allison et al. [ARA Collaboration], Astropart. Phys. 70, 62 (2015); M.-Y. Lu, C. Pfendner, and A. Shultz for the ARA Collaboration PoS(ICRC2017)966.

[59] J. Nam for the ANITA Collaboration, PoS(ICRC2017)952.

[60] P. W. Gorham et al. [ANITA Collaboration], Phys. Rev. D, 82, 022004 (2010). Phys. Rev. D 85, 049901(E) (2012).

[61] S. W. Barwick for the ARIANNA Collaboration, PoS(ICRC2017)1042

[62] A. G. Vieregg, K. Bechtol and A. Romero-Wolf, JCAP 02, 005 (2016); A.G. Vieregg et al. PoS(ICRC2017)1013.

[63] K. Fang et al. (GRAND Collaboration), PoS(ICRC2017)996. O. Martineau-Huyhn et al., EPJ Web of Conferences 135, 02001 (2017).

[64] J. D. Bray et al., Astropart. Phys. 77, 1 (2016).

[65] C. W. James et al., EPJ Web of Conferences 135, 04001 (2017).

[66] J. Alvarez-Muñiz, W. R. Carvalho, H. Schoorlemmer, K. Payet, A. Romero-Wolf and E. Zas, arXiv:1707.00334 [astro-ph.HE] submitted to Phys. Rev. D. and refs. therein.

[67] P. W. Gorham et al. (ANITA Collaboration), Phys. Rev. Lett. 117, 071101 (2016).

[68] A. Romero-Wolf for the ANITA Collaboration, PoS(ICRC2017)935.

[69] Q. Gou et al., PoS(ICRC2017)388.

[70] D. Ardouin, C. Cârloganu, D. Charrier, et al., Astropart. Phys. 34, 717 (2011).

[71] T. Liu et al., PoS(ICRC2017)234.

[72] J. Nam and T.C. Liu, PoS(ICRC2017)944. 